\title{
Fixed Point Theorems for Occasionally Weakly Compatible Mappings in Dislocated-Metric Spaces
}

\author{
Kastriot Zoto ${ }^{1, *}$, Elida Hoxha ${ }^{2}$, Panda Sumati Kumari ${ }^{3}$ \\ ${ }^{1}$ Department of Mathematics and Computer Sciences, Faculty of Natural Sciences, University of Gjirokastra Gjirokastra, Albania \\ ${ }^{2}$ Department of Mathematics, Faculty of Natural Sciences, University of Tirana; Tirana, Albania \\ ${ }^{3}$ KL University, Green Fields, Vaddeswaram, Guntur District, Andhra Pradesh, India \\ *Corresponding author: zotokastriot@yahoo.com
}

Received March 01, 2014; Revised April 03, 2014; Accepted April 10, 2014

\begin{abstract}
In this paper we prove some fixed point theorems for one and two pairs of selfmaps which are occasionally weakly compatible and satisfy a "max" and $(\psi-\phi)$ contractive conditions. Also, some existing results are derived as corollaries from theorems of this paper in the framework of dislocated metric spaces.
\end{abstract}

Keywords: occasionally weakly compatible, dislocated metric, contraction condition, common fixed point

Cite This Article: Kastriot Zoto, Elida Hoxha, and Panda Sumati Kumari, "Fixed Point Theorems for Occasionally Weakly Compatible Mappings in Dislocated-Metric Spaces." Turkish Journal of Analysis and Number Theory, vol. 2, no. 2 (2014): 37-41. doi: 10.12691/tjant-2-2-2.

\section{Introduction}

Hitzler and Seda in [18] introduced the concept of a dislocated metrics as a generalization of metrics where the self distance for any point need not to be equal to zero. They generalize the celebrated Banach contraction principle in dislocated metric spaces. Since then, many research papers have dealt with fixed point theory for single-valued mappings in dislocated metric spaces as a larger class than that of metric spaces (see, e.g., $[11,13,14,15,19,20,21]$.

Al-Thagafi and Shahzad [2] defined the concept of occasionally weakly compatible mappings which is more general than the concept of weakly compatible maps. Bhatt et al. [3] have given application of occasionally weakly compatible mappings in dynamical system. Motivated by the works of many authors for occasionally weakly compatible maps in metric spaces, in this paper we give some fixed point theorems for occasionally weakly compatible mappings satisfying $(\psi-\phi)$-weakly contractive condition in the setting of dislocated metric spaces. Our theorems unify and generalize various known results from metric spaces to dislocated metric spaces.

\section{Preliminaries}

Definition 2.1 [18] Let $X$ be a non-empty and let $d: X \times X \rightarrow R^{+}$be a function, called a distance function if for all $x, y, z \in X$, satisfies:

$$
\begin{gathered}
d_{1}: d(x, x)=0 \\
d_{2}: d(x, y)=d(y, x)=0 \Rightarrow x=y
\end{gathered}
$$

$$
\begin{gathered}
d_{3}: d(x, y)=d(y, x) \\
d_{4}: d(x, y) \leq d(x, z)+d(z, y) .
\end{gathered}
$$

If $d$ satisfies the condition $d_{1}-d_{4}$, then $d$ is called a metric on $X$. If it satisfies the conditions $d_{1}, d_{2}$ and $d_{4}$ it is called a quasi-metric. If $d$ satisfies conditions $d_{2}, d_{3}$ and $d_{4}$ it is called a dislocated metric (or simply $d$-metric). If $d$ satisfies only $d_{2}$ and $d_{4}$ then $d$ is called a dislocated quasi-metric (or simply dq-metric) on $X$. A nonempty set $X$ with dq-metric $d$, i. e., $(X, d)$ is called a dislocated quasi-metric space.

Definition 2.2 [18] A sequence $\left(x_{n}\right)$ in $d$-metric space $(X, d)$ is called Cauchy if for all $\varepsilon>0, \exists n_{0} \in N$ such that $\forall m, n \geq n_{0}, d\left(x_{m}, x_{n}\right)<\varepsilon$.

Definition 2.3 [18] A sequence $\left(x_{n}\right)$ dislocated converges or $d$-converges to $x$ if $\lim _{n \rightarrow \infty} d\left(x_{n}, x\right)=0$. In this case $x$ in called a $d$-limit of $\left(x_{n}\right)$ and we write $x_{n} \rightarrow x$.

Definition 2.4 [18] A $d$-metric space $(X, d)$ is complete if every Cauchy sequence in it is $d$-convergent.

Lemma 2.5 [18] Every subsequence of $d$-convergent sequence to a point $x_{0}$ is $d$-convergent to $x_{0}$.

Definition 2.6 [18] Let $(X, d)$ be a $d$-metric space. A mapping $T: X \rightarrow X$ is called contraction if there exists $0 \leq \lambda<1$ such that:

$$
d(T x, T y) \leq \lambda d(x, y) \text { for all } x, y \in X .
$$

Lemma 2.7 [18] Let $(X, d)$ be a $d$-metric space. If $f: X \rightarrow X$ is a contraction function, then $f^{n}\left(x_{0}\right)$ is a Cauchy sequence for each $x_{0} \in X$. 
Lemma 2.8 [18] $d$-limits in a $d$-metric space are unique. Definition 2.9 [9] Let $F$ and $S$ be mappings of a set $X$ into itself. Then, $F$ and $S$ are said to be weakly compatible if they commute at their coincidence point; that is $F x=S x$ for some $x \in X$ implies $S F x=F S x$.

Definition 2.10 [9] Two self-maps $f$ and $g$ of a set $X$ are occasionally weakly compatible (owc) iff there is a point $z$ in $X$ which is a coincidence point of $f$ and $g$ at which $f$ and $g$ commute.

Example 2.11 Let $X=[0,1]$ with dislocated metric $d(x, y)=x+y$. Define $f, g: X \rightarrow X$ by $f x=2 x$, $g x=x^{2}$. Then $C(f, g)=\{0,2\}, f(g(0))=g(f(0))$ and $f(g(2)) \neq g(f(2))$. Thus the pair $(f, g)$ is occasionally weakly compatible but not weakly compatible.

\section{Main Results}

After recalling some definitions and lemmas in dislocated metric space, we state the following theorems.

Theorem 3.1 Let $(X, d)$ be a dislocated-metric space and $f$ and $g$ are occasionally weakly compatible selfmappings of $X$, satisfying the condition:

$$
\begin{aligned}
& d(f x, f y) \\
\leq & c \max \left\{\begin{array}{l}
d(g x, g y), d(g x, f y), d(g y, f x), d(g y, f y), \\
d(g x, f x), d(g x, g x), d(g y, g y)
\end{array}\right\}
\end{aligned}
$$

for all $x, y \in X$ and $0 \leq c<\frac{1}{2}$. Then $f$ and $g$ have a unique common fixed point.

Proof. Since $f$ and $g$ are occasionally weakly compatible there exists a point $z$ in $X$ such that $f z=g z, f g z=g f z$. We claim that $f z$ is the unique common fixed point of $f$ and $g$. Let show that $f z$ is a fixed point of $f$. Consider:

$$
\begin{aligned}
& d(f z, f f z) \\
\leq & c \max \left\{\begin{array}{l}
d(g z, g f z), d(g z, f f z), d(g f z, f z), d(g f z, f f z), \\
d(g z, f z), d(g z, g z), d(g f z, g f z)
\end{array}\right\} \\
= & c \max \{d(f z, f f z), d(f f z, f f z), d(f z, f z)\} \\
\leq & 2 c d(f z, f f z)
\end{aligned}
$$

From this inequality and since $0 \leq c<\frac{1}{2}$, we have $d(f z, f f z)=0$. Thus $f f z=f z$ and from $f f z=f g z=g f z=f z$ we see that $f z$ is a common fixed point of $f$ and $g$.

Uniqueness. Suppose that $z$ and $v$ are two common fixed point of $f$ and $g$ such that $f z=g z=z$ and $f v=g v=v$ and $z \neq v$.

By condition (1) have:

$$
\begin{aligned}
& d(z, v)=d(f z, f v) \\
& \leq c \max \left\{\begin{array}{l}
d(g z, g v), d(g z, f v), d(g v, f z), d(g v, f v), \\
d(g z, f z), d(g z, g z), d(g v, g v)
\end{array}\right\} \\
& =c \max \{d(z, v), d(z, v), d(v, z), d(v, v), d(z, z)\} \\
& \leq 2 c d(v, z)
\end{aligned}
$$

since $0 \leq c<\frac{1}{2}$ we have $d(v, z)=0$. This implies $z=v$. Thus fixed point is unique.

Example 3.2 Let $X=[0,1]$ with dislocated metric $d(x, y)=x+y$. Define $f, g: X \rightarrow X$ by $f x=\frac{1}{4} x$ for $x \in[0,1]$ and $g x=\frac{x}{3}$ for $x \in[0,1)$ or $\frac{1}{4}$ for $x=1$. Then $C(f, g)=\{0,1\} \quad, \quad f(g(0))=g(f(0)) \quad$ and $f(g(1)) \neq g(f(1))$, so the pair $(f, g)$ is occasionally weakly compatible. Also for all $x, y \in X$ and $0 \leq c<\frac{1}{2}$ have,

$$
\begin{aligned}
d( & f x, f y)=\frac{1}{4}(x+y) \\
& \leq \frac{1}{3}(x+y) \\
& =d(g x, g y) \\
& \leq c \max \left\{\begin{array}{l}
d(g x, g y), d(g x, f y), d(g y, f x), d(g y, f y), \\
d(g x, f x), d(g x, g x), d(g y, g y)
\end{array}\right\}
\end{aligned}
$$

Thus all conditions of theorem are satisfied and 0 is the unique common fixed point of $f$ and $g$.

Corollary 3.3 Let $(X, d)$ be a dislocated-metric space and $f$ and $g$ are occasionally weakly compatible selfmappings of $X$, satisfying the condition:

$$
\begin{aligned}
& \qquad \begin{aligned}
d(f x, f y) \leq & \alpha d(g x, g y)+\beta d(g x, f y) \\
& +\gamma d(g y, f x)+\delta d(g y, f y) \\
& +\eta d(g x, f x)+\mu d(g x, g x)+\theta d(g y, g y)
\end{aligned} \\
& \text { for all } \quad x, y \in X \quad \text { and nonnegative constant } \\
& \alpha, \beta, \gamma, \delta, \eta, \mu, \theta \text { with } 0 \leq \alpha+\beta+\gamma+\delta+\eta+\mu+\theta<\frac{1}{2} .
\end{aligned}
$$

Then $f$ and $g$ have a unique common fixed point.

Proof. This theorem can be obtained as corollary of theorem 3.1 since we have that;

$$
\begin{aligned}
& d(f x, f y) \leq \alpha d(f x, g y)+\beta d(g x, f y) \\
&+\gamma d(g x, g y)+\delta d(g y, f y) \\
&+\eta d(g x, f x)+\mu d(g x, g x)+\theta d(g y, g y) \\
& \leq(\alpha+\beta+\gamma+\delta+\eta+\mu+\theta) \\
& \max \left\{\begin{array}{l}
d(f x, g y), d(g x, f y), d(g x, g y), d(g y, f y), \\
d(g x, f x), d(g x, g x), d(g y, g y)
\end{array}\right\}
\end{aligned}
$$


for all $x, y \in X$ and constant $\alpha, \beta, \gamma, \delta, \eta, \mu, \theta$ non negative with $0 \leq \alpha+\beta+\gamma+\delta+\eta+\mu+\theta<\frac{1}{2}$.

For the following theorem which involve two pairs of self mappings each owc, we use the class of function $\Psi ; \Phi$ where $\Psi=\{\psi:[0, \infty) \rightarrow[0, \infty)\}$ such that $\psi$ is a continuous non decreasing with $\psi(t)=0$ iff $t=0$, $\Phi=\{\phi:[0, \infty) \rightarrow[0, \infty)\}$ such that $\phi$ is a continuous function with $\phi(t)=0$ iff $t=0$ and denote

$$
M(x, y)=\max \left\{\begin{array}{l}
d(S x, T y), d(S x, G y), d(T y, F x), \\
\frac{k}{4}[d(S x, F x)+d(T y, G y)]
\end{array}\right\} .
$$

Fixed point results that we are proving can be considered as continuation or generalization of many results given by $[11,13,14,16]$.

Theorem3.4 Let $(X, d)$ be a dislocated metric space and $F, G, S$ and $T$ be self-mappings of $X$. The pairs $(F, S)$ and $(G, T)$ are owc, and satisfy the condition:

$$
\psi(d(F x, G y)) \leq \psi(M(x, y))-\phi(M(x, y))
$$

for all $x, y \in X$ and $0<k<\frac{1}{4}$ where $\psi \in \Psi, \phi \in \Phi$.

Then there exists a unique common fixed point of $F, G, S$ and $T$.

Proof. Since the pairs $(F, S)$ and $(G, T)$ are owc, there are points $x, y \in X$ such that $F x=S x$ and $F S x=S F x$, $G y=T y$ and $G T Y=T G y$. We claim that $F x=G y$. Consider that

$$
\begin{aligned}
M(x, y) & =\max \left\{\begin{array}{l}
d(S x, T y), d(S x, G y), d(F x, T y), \\
\frac{k}{4}[d(S x, F x)+d(T y, G y)]
\end{array}\right\} \\
& =\max \left\{\begin{array}{l}
d(F x, G y), d(F x, G y), d(F x, G y), \\
\frac{k}{4}[d(F x, F x)+d(G y, G y)]
\end{array}\right\} \\
& =d(F x, G y)
\end{aligned}
$$

By the condition of theorem have:

$$
\psi(d(F x, G y)) \leq \psi(d(F x, G y))-\phi(d(F x, G y))
$$

This inequality is a contradiction unless $d(F x, G y)=0$, thus we have $F x=G y$, i.e. $F x=S x=G y=T y$. Firstly observe that

$$
\begin{aligned}
M(F x, y) & =\max \left\{\begin{array}{l}
d(S F x, T y), d(S F x, G y), d(F F x, T y), \\
\frac{k}{4}[d(F F x, G y)+d(T y, G y)]
\end{array}\right\} \\
& =\max \left\{\begin{array}{l}
d(F F x, G y), d(F F x, G y), d(F F x, G y), \\
\frac{k}{4}[d(F F x, G y)+d(G y, G y)]
\end{array}\right\} \\
& =d(F F x, F x)
\end{aligned}
$$

Suppose that $F^{2} x \neq F x$, then inequality (2) gives:

$$
\begin{aligned}
\psi\left(d\left(F^{2} x, F x\right)\right) & =\psi(d(F F x, G y)) \\
& \leq \psi(M(F x, y))-\phi(M(F x, y)) \\
& =\psi(M(F F x, F x))-\phi(M(F F x, F x))
\end{aligned}
$$

which is a contradiction. Hence $d(F F x, F x)=0$ and so $F F x=F x=S F x$.

Thus $F x$ is a fixed point of $F$ and $S$.

In the same way, we observe that

$$
\begin{aligned}
M(x, G y) & =\max \left\{\begin{array}{l}
d(S x, T G y), d(S x, G G y), d(F x, T G y), \\
\frac{k}{4}[d(S x, F x)+d(T G y, G G y)]
\end{array}\right\} \\
& =\max \left\{\begin{array}{l}
d(G y, G G y), d(G y, G G y), d(G y, G G y), \\
\frac{k}{4}[d(G y, G y)+d(G G y, G G y)]
\end{array}\right\} \\
& =d(G y, G G y)
\end{aligned}
$$

If suppose that $G G y \neq G y$, from condition (2) we have,

$$
\begin{aligned}
\psi(d(G G y, G y)) & =\psi(d(F x, G G y)) \\
& \leq \psi(M(x, G y))-\phi(M(x, G y)) \\
& =\psi(d(G G y, G y))-\phi(d(G G y, G y))
\end{aligned}
$$

which is a contradiction, unless $d(G y, G G y)=0$. Therefore, we get $G G y=G y=T G y$, and $F x=S x=G y=T y$ is a common fixed point of $F, G, S$ and $T$.

Uniqueness. If we assume that there exists two common fixed points $u$ and $z$ of $F, G, S$ and $T$. For $u \neq z$ again from the condition of theorem we get

$$
\begin{aligned}
M(u, z) & =\max \left\{\begin{array}{l}
d(S u, T z), d(S u, G z), d(F u, T z), \\
\frac{k}{4}[d(S u, F u)+d(T z, G z)]
\end{array}\right\} \\
& =d(u, z)
\end{aligned}
$$

So

$$
\begin{aligned}
\psi(d(u, z)) & =\psi(d(F u, G z)) \\
& \leq \psi(M(u, z))-\phi(M(u, z)) \\
& =\psi(d(u, z))-\phi(d(u, z))
\end{aligned}
$$

which is a contradiction unless $d(u, z)=0$ and as a result $u=z$. Therefore $u$ is the unique common fixed point of $F, G, S$ and $T$.

Corollary 3.5 Let $(X, d)$ be a dislocated metric space and $F, S$ and $T$ be self-mappings of $X$. The pairs $(F, S)$ and $(F, T)$ are owc, and satisfy the condition:

$$
\psi(d(F x, F y)) \leq \psi(M(x, y))-\phi(M(x, y))
$$

for all $x, y \in X$ and $0<k<\frac{1}{4}$ where $\psi \in \Psi, \phi \in \Phi$. 
Then there exists a unique common fixed point of $F, S$ and $T$.

Proof. This is clear if in theorem3.4 we put $G=F$.

Corollary 3.6 Let $(X, d)$ be a dislocated-metric space and $F$ and $G$ are occasionally weakly compatible selfmappings of $X$, satisfying the condition:

$$
\psi(d(F x, F y)) \leq \psi(M(x, y))-\phi(M(x, y))
$$

for all $x, y \in X$ and $0 \leq 4 k<1$ and functions $\psi \in \Psi$, $\phi \in \Phi$. Then $F$ and $G$ have a unique common fixed point. Proof. The proof is taken from theorem 3.4 if we take in it $S=T=i$ (identity map)

Example 3.7 Let $X=[0,1]$ with dislocated metric $d(x, y)=x+y$. Define $f, g: X \rightarrow X$ by $f x=x^{2}$, $g x=2 x$. Then $C(f, g)=\{0,2\}, f(g(0))=g(f(0))$ and $f(g(2)) \neq g(f(2))$. Thus the pair $(f, g)$ is occasionally weakly compatible but not weakly compatible, and for functions $\psi ; \phi$ as $\psi(t)=\frac{t}{2}$ and $\phi(t)=\frac{t}{4}$ we observe that,

$$
\begin{aligned}
\psi(d(f x, f y)) & =\frac{1}{2} d(f x, f y)=\frac{1}{2}\left(x^{2}+y^{2}\right) \leq \frac{1}{2}(x+y)= \\
& =\frac{1}{4}(2 x+2 y)=\frac{1}{4} d(g x, g y) \leq \frac{1}{4} M(x, y)= \\
& =\frac{1}{2} M(x, y)-\frac{1}{4} M(x, y) \\
& =\psi(M(x, y))-\phi(M(x, y))
\end{aligned}
$$

for all $x, y \in X$.

Thus all conditions of theorem hold and 0 is the unique common fixed point of $f$ and $g$.

Corollary 3.8 Let $(X, d)$ be a dislocated metric space and $F, G, S$ and $T$ be self-mappings of $X$. The pairs $(F, S)$ and $(G, T)$ are owc, and satisfy the condition:

$$
d(F x, G y) \leq M(x, y)-\phi(M(x, y))
$$

for all $x, y \in X$ and $0<k<\frac{1}{4}$ where $\phi \in \Phi$.

Then there exists a unique common fixed point of $F, G, S$ and $T$.

This corollary is taken from theorem 3.4 If we take the function $\psi$ as $\psi(t)=t$.

Corollary 3.9 Let $(X, d)$ be a dislocated-metric space and $F$ and $S$ are occasionally weakly compatible selfmappings of $X$, satisfying the condition:

$$
\psi(d(F x, F y)) \leq \psi(M(x, y))-\phi(M(x, y))
$$

for all $x, y \in X$ and $0 \leq k<\frac{1}{4}$ where $\psi \in \Psi, \phi \in \Phi$. Then $F$ and $S$ have a unique common fixed point.

This corollary is taken from theorem if we put in it $G=F$ and $T=S$.
Corollary 3.10 Let $(X, d)$ be a dislocated metric space and $F, G, S$ and $T$ be self-mappings of $X$. The pairs $(F, S)$ and $(G, T)$ are owc, and satisfy the condition:

$$
d(F x, G y) \leq r M(x, y)
$$

for all $x, y \in X, 0 \leq r<1$ and $0<k<\frac{1}{4}$.

Then there exists a unique common fixed point of $F, G, S$ and $T$.

This corollary is taken from above corollary3.8 if we take in it the function $\phi(t)=(1-r) t$ for $0 \leq r<1$.

Let be the class $L$ of functions $p: R^{+} \rightarrow R^{+}$which are Lebesgue integrable functions and summable nonnegative such that $\int_{0}^{\varepsilon} p(t) d t>0$ for each $\varepsilon>0$. Now we give the following fixed point theorems for occasionally weakly compatible mappings satisfying contractive conditions of integral type.

Theorem 3.11 Let $(X, d)$ be a dislocated metric space and $F, G, S$ and $T$ be self-mappings of $X$. The pairs $(F, S)$ and $(G, T)$ are owc, and satisfy the condition:

$$
\int_{0}^{d(F x, G y)} p(r) d r \leq \int_{0}^{M(x, y)} p(r) d r-\int_{0}^{M(x, y)} h(r) d r
$$

for all $x, y \in X$ and $0<k<\frac{1}{4}$ where $p, h \in L$.

Then $F, G, S$ and $T$ have a unique common fixed point. Proof. If we take $\psi(r)=\int_{0}^{r} p(s) d s \quad$ and $\phi(r)=\int_{0}^{r} h(s) d s$ then we see that the functions $\psi, \phi$ are functions from $\Psi ; \Phi$. So on this conditions, we can use theorem 3.4 and the self mappings $F, G, S$ and $T$ have a unique common fixed point.

Corollary 3.12 Let $(X, d)$ be a dislocated metric space and $F, G, S$ and $T$ be self-mappings of $X$. The pairs $(F, S)$ and $(G, T)$ are owc, and satisfy the condition:

$$
\int_{0}^{d(F x, G y)} p(s) d s \leq \lambda \int_{0}^{M(x, y)} p(s) d s
$$

for all $x, y \in X, 0<\lambda<1$ and $0<k<\frac{1}{4}$ where $p \in L$.

Then $F, G, S$ and $T$ have a unique common fixed point.

Proof. If we take $h(r)=(1-\lambda) p(r)$ then from theorem 3.11 we conclude that $F, G, S$ and $T$ have a unique common fixed point.

Remark 3.13 These theorems are an extension of many results on fixed point given in dislocated metric spaces by authors $[11,13,14,15,16,17,19,22,23]$ for occasionally weakly compatible mappings without imposing conditions on the space or mappings such as completeness, closedness and continuity. 


\section{Acknowledgements}

The authors are thankful to the editor and referees, for their valuable suggestions for the improvement of the paper.

\section{References}

[1] M. Aamri and D. El Moutawakil, Some new common fixed point theorems under strict contractive conditions, J. Math. Anal. Appl., 270 (2002), 181-188.

[2] M.A. Al-Thagafi and N. Shahzad, Generalized I-nonexpansive selfmaps and invariant approximations, Acta Math. Sinica, 24(5) (2008), 867-876.

[3] A. Bhatt, H. Chandra and D.R. Sahu, Common fixed point theorems for occasionally weakly compatible mappings under relaxed conditions, Nonlinear Analysis, 73(1) (2010), 176-182.

[4] H. Chandra and A. Bhatt, Fixed point theorems for occasionally weakly compatible maps in probabilistic semi-metric space, Int. J. Math. Anal., 3(12) (2009), 563-570.

[5] S. Chauhan and B. D. Pant, Common fixed point theorems for occasionally weakly compatible mappings using implicit relation, Journal of the Indian Math. Soc., 77(1-4) (2010) 13-21.

[6] I. Beg, M. Abbas, Coincidence point and invariant approximation for mappings satisfying generalized weak contractive condition, Fixed Point Theory and Applications, 2006, Article ID 74503, pages 1-7.

[7] S. S. Chauhan and K. Utreja, A common fixed point theorem in dislocated metric space, International Journal of Engineering and Innovative Technology (IJEIT), vol 3. Issue1. July 2013.

[8] M. Abbas and B. E. Rhoades, Common fixed point theorems for occasionally weakly compatible mappings satisfying a generalized contractive condition Mathematical Communications 13(2008), 295-301 295.

[9] G. Jungck, B. E. Rhoades, Fixed point theorems for occasionally weakly compatible mappings, Fixed Point Theory 7(2006), 287296.
[10] G. Jungck, B. E. Rhoades, Fixed point theorems for occasionally weakly compatible mappings, Fixed Point Theory 9(2008), 383384 (erratum).

[11] K. Zoto and E. Hoxha, Fixed point theorems in dislocated and dislocated quasi-metric spaces.Journal of advanced study in topology vol4.nr.3, 2012.

[12] A. Isufati. Fixed point theorems in dislocated quasi-metric space. Appl. Math. Sci., 4(5): 217-223, 2010.

[13] Kumari et al, Common fixed point theorems on weakly compatible maps on dislocated metric spaces. Mathematical Sciences 2012 6:71.

[14] C. T. Aage and J. N. Salunke. The results on fixed points in dislocated and dislocated quasi-metric space. Appl. Math. Sci., 2(59): 2941-2948, 2008

[15] K. Zoto, Weakly compatible mappings and fixed points in dislocated -metric spaces, International journal of mathematical archive, vol. 4 (6), 2013, 131-137.

[16] K. Jha and D. Panthi, A Common Fixed Point Theorem in Dislocated Metric Space, Appl. Math. Sci., vol. 6, 2012, no. 91, 4497-4503.

[17] K. P. R. Rao and P. Rangaswamy, Common Fixed Point Theorem for Four Mappings in Dislocated Quasi-Metric Space, The Nepali Math. Sci. Report, 30 (1-2), 2010, 70-75.

[18] P. Hitzler and A. K. Seda. Dislocated topologies. J. Electr. Engin., 51(12/S): 3: 7, 2000.

[19] P. Hitzler: Generalized metrics and topology in logic programming semantics, Ph. D Thesis, School of Mathematics, Applied Mathematics and Statistics, National University Ireland, University college Cork, 2001.

[20] R. Shrivastava, Z.K. Ansari and M. Sharma. Some results on Fixed Points in Dislocated and Dislocated Quasi-Metric Spaces. Journal of Advanced Studies in Topology; Vol. 3, No.1, 2012,

[21] I. R. Sarma and P. S. Kumari, "On dislocated metric spaces," International Journal of Mathematical Archive, vol. 3, no. 1, pp. 72-77, 2012.

[22] Hussain et al, Common fixed point results for weak contractive mappings in ordered b-dislocated metric spaces with applications. Journal of Inequalities and Applications 2013, 2013: 486.

[23] P. S. Kumari, Some fixed point theorems in generalized dislocated metric spaces, Mathematical Theory and Modeling, 1 (4), 16-22. 